\title{
Electrical stimulation-fracture treatment: new insights into the underlying mechanisms
}

\author{
Mit Balvantray Bhavsar ${ }^{1}$ (D), Liudmila Leppik¹, Karla Mychellyne Costa Oliveira ${ }^{1}$ \& John H \\ Barker*,1 \\ ${ }^{1}$ Frankfurt Initiative for Regenerative Medicine, JW Goethe-University, Frankfurt am Main, Germany \\ *Author for correspondence: Tel.: +49 0696705 9240; jhb121654@gmail.com
}

First draft submitted: 29 March 2019; Accepted for publication: 9 April 2019; Published online: 6 June 2019

Non healing fractures, caused by injury or resection of infected bone or tumors, are a major challenge in trauma and orthopedic surgery. Bone is one of the few tissues whose natural response to injury is to regenerate, thus restoring its form and function back to its original state, instead of healing and forming a scar. Whether and to what degree bone regenerates or heals and scars is dictated by many local and systemic factors, including species, age, type and extent of injury, and physical and chemical signals. Among the latter, electrochemical signals are known to play an important role in regulating regeneration and embryonic development.

Cell function during embryonic development and regeneration is regulated by electrochemical signals that result from positively and negatively charged ions passing through their membranes. This passage of charged ions generates transmembrane voltage gradients or $\mathrm{V}_{\text {mem }}$. The $\mathrm{V}_{\text {mem }}$ values on the surface of cells that are highly proliferative, like embryonic stem and cancer cells tend to be more positive and are depolarized, whereas $V_{\text {mem }}$ values of cells in low proliferative states, like neurons, fibroblasts, skeletal muscle and fat cells are more negative, and are hyperpolarized [15]. During embryonic development $V_{\text {mem }}$ values are in constant flux, and these changes have been shown to play an important role in regulating growth throughout development. This was clearly demonstrated in a series of landmark experiments conducted by Shi and Borgens in which they measured distinct bioelectric patterns on the surface of chick embryos during development. They found that by short circuiting these bioelectric current flows, with a copper wire implanted beneath the skin, newborn chicks hatched without lower extremities [6]. The presence of bioelectric signals during regeneration was demonstrated, by the same investigators, in a separate set of experiments. They measured endogenous bioelectric current emanating from the stumps of amputated, regenerating newt limbs, and found distinct patterns of current irradiating from the limb stumps. In these experiments they describe higher intensities of bioelectric current 4 days after amputation that gradually subsided over the course of a week [7].

Externally applied electrical stimulation (EStim) has been used successfully in clinical settings to improve bone healing for many years. The earliest mention of using EStim to treat fractures in patients was in the mid-1800s in which Garrat used metallic needles to deliver EStim directly into broken bones, and reported improved healing [8]. More recently EStim has been used in clinical settings, primarily as an adjunct to other treatments, to treat recalcitrant fractures [9]. In these cases, EStim is administered using three different approaches; direct current, pulsed electromagnetic field (PEMF) and capacitive coupled (CC). Direct current EStim is applied via a surgically implanted EStim power source and electrodes, at intensities between 10 and $100 \mu \mathrm{A}$ of current [10]. CC and pulsed PEMF are both administered externally. In CC, an alternating voltage is applied via electrodes placed on the skin on opposite sides of a fracture, generating an electrical field of 0.1-20 G [11]. In PEMF, alternating currents, in an electric coil placed over the fracture site, generate a pulsed electromagnetic field, ranging between 3 and $10 \mathrm{~V}$ peak-to-peak [12].

In spite of demonstrated success promoting bone healing, only recently have we begun to understand the mechanisms by which EStim produces this pro-healing effect. The results of several recently published in vitro studies suggest that EStim's pro-healing effect is due, at least in part, to its effect on the behavior of bone-forming stem cells. Along these lines, we, and others have shown that EStim improves bone stem cell migration, proliferation, alignment, differentiation and attachment to scaffold materials [13-16]. In addition to these effects on cell behavior we and others have also shown that exposing these cells to externally applied EStim increases mineralization, extracellular matrix deposition and enhanced expression of several osteogenic genes [17,18], all behaviors/functions

Future Medicine 
known to be central in both bone healing and regeneration. In in vitro studies, we exposed bone-forming cells, in culture to externally applied EStim and observed significant increases in metabolic activity and calcium deposition (osteogenic differentiation). We found that exposure to EStim caused the highest metabolic activity at 3 days of culture, and that EStim caused distinct changes in cell morphology and increased osteogenic differentiation at 7 and 14 days. These changes in cell behavior were accompanied by important changes in osteogenic gene expression patterns, with a significant increase in mRNA levels of Runx2, osteopontin and Col1A2 [18,19].

In other in vitro experiments, Wiesmann et al. [20] reported, that stimulating osteoblasts with CC EStim accelerated mineralization, and speculated the cause to be 'electrochemical processes' occurring during EStim. In a separate study, Nguyen et al. [21] showed that EStim changed calcium distribution within Matrigel and Schwann cells seeded onto gels, a week after stimulation, demonstrating that EStim can have a long-term effect on extracellular matrix deposition. Fredericks et al. [22] showed, for the first time, that DC EStim caused a sustained increase of multiple osteogenic genes, suggesting that the biologic mechanism for the observed increase in the rate and extent of bone formation, was mediated by the upregulation of these osteoinductive factors.

In a separate set of in-vivo studies, in a rat limb amputation model, we found that EStim stimulates bone marrow-derived stem/progenitor cells to generate highly vascularized osseocartilaginous centers in the zone of injury. We observed that the bone marrow cavity of EStim treated limb stumps remained open for 7 days, whereas in non-EStim sham and control animals the cavity was closed with a thin layer of newly formed fibrous scar-like tissue at day 7 [23]. We hypothesized that this delay in closure of the bone marrow cavity was due to EStim shifting the 'balance' from healing and scarring (seen in control stumps) toward continued proliferation and regeneration. This is an important observation that may provide a hint into the mechanisms by which EStim promotes bone regeneration over healing and scarring.

In another set of in vivo experiments, this time in a rat large bone defect model, we exposed defects treated with MSC and scaffold material with EStim and measured the rate and quality of healing [24]. As in our limb amputation experiments, we found that EStim further improved the healing, already provided by the stem cell + scaffold treatment. Of note, by 8 weeks, we found that defects in the control and sham animals contained greater amounts of newly formed fibrous tissue and less new bone and cartilage, than in the EStim-treated defects. In the latter, large areas of the defect were replaced with newly formed bone and cartilage. Although at this 8-week end point, complete bone healing was not observed in any of the EStim-treated samples, significant new bone growth into the center of the defect was evident. This observation supported our hypothesize that EStim's positive effect on bone healing results from reducing fibrous tissue formation, in favor of new bone and cartilage formation. In other words, shifting the balance from healing and scarring toward regeneration.

The exciting new field, and recent advancements in regenerative medicine technologies, hold tremendous potential for completely changing how we practice medicine. For the first time we may be able to actually cure disease, rather than just treat the symptoms. Endogenous bioelectric signals are known to play an important role in regeneration. Externally applied electrical stimulation can be used to manipulate these bioelectric signals to promote regenerative behaviors and functions in cells. A better understanding of the mechanisms by which endogenous and externally applied electrical currents regulate regenerative processes is needed. The many tools we now have at our disposition for probing these mechanisms, at a cellular and molecular level, are starting to reveal previously unknown secrets that could be used to develop powerful new approaches for unleashing the full potential of regenerative medicine.

\section{Financial \& competing interests disclosure}

The authors have no relevant affiliations or financial involvement with any organization or entity with a financial interest in or financial conflict with the subject matter or materials discussed in the manuscript. This includes employment, consultancies, honoraria, stock ownership or options, expert testimony, grants or patents received or pending, or royalties.

No writing assistance was utilized in the production of this manuscript.

\section{References}

1. Stillwell EF, Cone CM, Cone CD. Stimulation of DNA synthesis in CNS neurons by sustained depolarization, Nat. New Biol. 246(152), 110-111 (1973).

2. Levin M. Molecular bioelectricity in developmental biology: new tools and recent discoveries: control of cell behavior and pattern formation by transmembrane potential gradients. BioEssays 34(3), 205-217 (2012). 
3. Sundelacruz S, Levin M, Kaplan DL. Membrane potential controls adipogenic and osteogenic differentiation of mesenchymal stem cells. PLoS ONE 3 e3737 (2008).

4. Sundelacruz S, Levin M, Kaplan DL. Role of membrane potential in the regulation of cell proliferation and differentiation. Stem Cell Rev. 5(3), 231-246 (2009).

5. Sundelacruz S, Moody AT, Levin M, Kaplan DL. Membrane potential depolarization alters calcium flux and phosphate signaling during osteogenic differentiation of human mesenchymal stem cells. Bioeletricity 1(1),56-66 (2019).

6. Shi R, Borgens RB. Three dimensional gradients of voltage during development of the nervous system as invisible coordinates for the establishment of the embryonic pattern. Dev. Dynamics 202(2), 101-114 (1995).

7. Borgens RB, Vanable JW Jr, Jaffe LF. Bioelectricity and regeneration: large currents leave the stumps of regenerating newt limbs. Proc. Natl Acad. Sci. USA 74(10), 4528-4532 (1977).

8. Garrat AC. Electrophysiology and electrotherapeutics. Ticknor and Fields Boston, MA, USA (1860).

9. Griffin M, Bayat A. Electrical stimulation in bone healing: critical analysis by evaluating levels of evidence. Eplasty 11, 303-353 (2011)

10. Kuzyk PR, Schemitsch EH. The science of electrical stimulation therapy for fracture healing. Indian J. Orthop. 43(2), 127-131 (2009).

11. Chalidis B, Sachinis N, Assiotis A, Maccauro G. Stimulation of bone formation and fracture healing with pulsed electromagnetic fields: biologic responses and clinical implications. Int. J. Immunopathol. Pharmacol. 24(1 Suppl 2), 17-20 (2011).

12. Aaron RK, Boyan BD, Ciombor DM, Schwartz Z, Simon BJ. Stimulation of growth factor synthesis by electric and electromagnetic fields. Clin. Orthop. Relat. Res. 419, 30-37 (2004).

13. Gittens RA, Olivares-Navarrete R, Rettew R et al. Electrical polarization of titanium surfaces for the enhancement of osteoblast differentiation. Bioelectromagnetics 34(8), 599-612 (2013).

14. Hu WW, Hsu YT, Cheng YC et al. Electrical stimulation to promote osteogenesis using conductive polypyrrole films. Mater. Sci. Eng. C. 37, 28-36 (2014).

15. Mobini S, Leppik L, Barker JH. Direct current electrical stimulation chamber for treating cells in vitro. Biotechniques 60(2), 95-98 (2016).

16. Kwon HJ, Lee GS, Chun H. Electrical stimulation drives chondrogenesis of mesenchymal stem cells in the absence of exogenous growth factors. Sci. Rep. 6, 39302 (2016).

17. Zhang J, Li M, Kang ET, Neoh KG. Electrical stimulation of adipose-derived mesenchymal stem cells in conductive scaffolds and the roles of voltage-gated ion channels. Acta Biomater. 32, 46-56 (2016).

18. Mobini S, Leppik L, Thottakkattumana PV, Barker JH. In vitro effect of direct current electrical stimulation on rat mesenchymal stem cells. PeerJ 5, e2821 (2017).

19. Eischen-Loges M, Oliveira KMC, Bhavsar MB, Barker JH, Leppik L. Pretreating mesenchymal stem cells with electrical stimulation causes sustained long-lasting pro-osteogenic effects. PeerJ 6, e4959 (2018).

20. Wiesmann H, Hartig M, Stratmann U, Meyer U, Joos U. Electrical stimulation influences mineral formation of osteoblast-like cells in vitro. Biochim. Biophys. Acta 1538(1), 28-37 (2001).

21. Nguyen HT, Wei C, Chow JK, Nguy L, Nguyen HK, Schmidt CE. Electric field stimulation through a substrate influences Schwann cell and extracellular matrix structure. J. Neural Eng. 10(4), 46011 (2013).

22. Fredericks DC, Smucker J, Petersen EB et al. Effects of direct current electrical stimulation on gene expression of osteopromotive factors in a posterolateral spinal fusion model. Spine 32(2), 174-81 (2007).

23. Leppik LP, Froemel D, Slavici A et al. Effects of electrical stimulation on rat limb regeneration, a new look at an old model. Sci. Rep. 5, 18353 (2015).

24. Leppik L, Zhihua H, Mobini S et al. Combining electrical stimulation and tissue engineering to treat large bone defects in a rat model. Sci. Rep. 8(1), 6307 (2018). 